\title{
The HPLC-double-cluster pattern of some Mycobacterium gordonae strains is due to their dicarboxy-mycolate content
}

\author{
José Astola, ${ }^{1}$ Manuel Muñoz, ${ }^{1}$ Marco Sempere, ${ }^{2}$ Pere Coll, ${ }^{1,3}$ \\ Marina Luquin ${ }^{1}$ and Pedro L. Valero-Guillén ${ }^{4}$
}

\footnotetext{
1 Departament de Genètica i Microbiologia, Universitat Autònoma de Barcelona, Spain

2 Hospital Costa del Sol, Marbella, Málaga, Spain

3 Hospital de la Santa Creu i Sant Pau, Barcelona, Spain

4 Departamento de Genética y Microbiología, Facultad de Medicina, Universidad de Murcia, Aptdo. 4012, Campus Universitario de Espinardo, 30100 Murcia, Spain
}

Author for correspondence: Pedro L. Valero-Guillén. Tel:+34 968 360953. Fax: +34 968360950. e-mail: plvalero@um.es

The mycolic acids of several strains of Mycobacterium gordonae were examined by chromatographic and spectroscopic techniques. Both HPLC and TLC revealed two patterns of mycolates among the $M$. gordonae strains studied. As determined by TLC, one pattern was composed of $\alpha$-, methoxy- and keto-mycolates; the other was composed of these mycolates plus an additional component, which was identified as dicarboxy-mycolates. The dicarboxymycolates were only found in those $M$. gordonae strains that displayed a so-called HPLC-double-cluster pattern. Detailed structural analyses of the dicarboxy-mycolates indicated that these compounds contained predominantly 61-65 carbon atoms ( $C_{63}$ was the major component) and a trans-1,2disubstituted cyclopropane ring. Thus, the dicarboxy-mycolate content of strains of $M$. gordonae determines their HPLC pattern. In spite of the differences in their HPLC patterns, and although they belonged to different PCR-restriction length polymorphism clusters, all of the $M$. gordonae strains examined in this study were closely related on the basis of the structural features of their $\alpha$-, keto- and methoxy-mycolates; the predominant $\alpha$-mycolates contained two cis-1,2-disubstituted cyclopropane rings, the major keto-mycolates contained a trans-1,2-disubstituted cyclopropane ring and the methoxy-mycolates contained one cis- or one trans-1,2-disubstituted cyclopropane ring. It is noteworthy that the strains containing dicarboxymycolates also displayed significant amounts of $\alpha$-mycolates that contained one cis-1,2-disubstituted cyclopropane ring and one cis double bond. The results obtained in this study demonstrate heterogeneity among $M$. gordonae strains.

Keywords: mycolic acids, high-performance liquid chromatography, M. gordonae

\section{INTRODUCTION}

Mycobacterium gordonae is a slow-growing scotochromogenic species of Mycobacterium, which is widely distributed in natural water sources and soils (Wayne \& Kubica, 1986). It has been found in bottled table waters (Papapetropoulou et al., 1997) and is the most common mycobacterial isolate from swimming-pool environments (Leoni et al., 1999), probably due to its resistance to chlorine disinfection (Le Dantec et al., 2002). Several

Abbreviations: El-MS, electron-impact MS; FAB-MS, fast-atom bombardment MS; Gen-Probe, Gen-Probe Rapid Diagnostic System; PRA, PCRrestriction length polymorphism analysis.
M. gordonae-associated infections have been observed in endoscopy patients; these are thought to have been due to the use of improperly cleaned or disinfected instruments (Vogiatzakis et al., 1998). Although M. gordonae is considered to be non-pathogenic, there have been some well-documented cases of infections caused by this organism in patients with underlying conditions, e.g. AIDS or cancer (Bernard et al., 1992; Clague et al., 1985; London et al., 1988; Rusconi et al., 1997).

Molecular-based methodologies for the rapid identification of M. gordonae isolates have revealed genetic variability within this species. In contrast to other mycobacteria, which show conservation of rDNA sequences at the species level, M. gordonae strains exhibit 
variation within a region of their rDNA; this region is a common target for diagnostic species-specific probes (Kirschner \& Böttger, 1992). The microheterogeneity observed among strains of $M$. gordonae could be the reason for the hybridization problems reported by Walton \& Valesco (1991) when using the Gen-Probe Rapid Diagnostic System (Gen-Probe). Studies using other molecular-based identification methods, such as PCR-restriction length polymorphism analysis (PRA), have concluded that $M$. gordonae is the most heterogenous member of the genus Mycobacterium studied to date, generating several closely related subclusters upon analysis of strain data (Plikaytis et al., 1992; Telenti et al. 1993).

Mycolic acids are high-molecular-mass 3-hydroxy, 2alkyl-branched fatty acids found in all Mycobacterium species. The different structural types of mycolic acids include the so-called $\alpha$-mycolates and the mycolates which have other oxygen functions (i.e. the keto-, methoxy-, dicarboxy-, epoxy- and $\omega-1$ methoxy-mycolates) in addition to the 3-hydroxy acid unit (Minnikin, 1982; Minnikin et al., 1982; Luquin et al., 1990). The use of TLC to analyse mycolic acid methyl esters allowed the different structural types of mycolic acids to be separated. The mycolate patterns obtained upon separation of mycolic acid methyl esters by TLC have been widely used in the classification and identification of mycobacteria (Daffé et al., 1983; Minnikin et al., 1984; Luquin et al., 1991; Valero-Guillén et al., 1985). M. gordonae exhibits a TLC mycolate pattern characterized by the presence of $\alpha$-, methoxy- and keto-mycolates. Analysis of mycolic acids by HPLC has permitted a large proportion of mycobacterial species to be identified by their unique and reproducible chromatographic patterns (Butler \& Guthertz, 2001). However, M. gordonae isolates have been shown to produce two different HPLC mycolic acid patterns - a single-cluster pattern, which is the most common pattern, and a double-cluster pattern (Cage, 1992; Butler et al., 1991). To date, no studies have been done to correlate these patterns with differences in mycolate production between strains of M. gordonae or to identify the compounds responsible for the second cluster of peaks seen in the double-cluster pattern.

The aim of this work was to elucidate the lipidic compounds of M. gordonae that give rise to the HPLCdouble-cluster pattern. We studied five clinical isolates of M. gordonae that produced the HPLC-double-cluster pattern; for comparative purposes, we also studied two reference strains of $M$. gordonae. The lipidic component responsible for the second peak in the double-cluster pattern was purified and identified by spectrometric methods as dicarboxy-mycolate, a mycolic acid that has not been described in M. gordonae until now.

\section{METHODS}

Strains, cultivation and identification. Five strains from clinical samples (CR-178, CL-333 and CL-361, from sputa; CL-416C, from an alveolar lavage; CL-554, from a broncho- aspirate) were examined, together with $M$. gordonae strains ATCC $14470^{\mathrm{T}}$ (HPLC-single-cluster pattern) and ATCC 35759 (HPLC-double-cluster pattern). All of the strains were grown on Sauton's medium at $32{ }^{\circ} \mathrm{C}$ for 6 weeks.

Identification of the strains was done by using standard biochemical methods (Tsukamura, 1967) and Gen-Probe for $M$. gordonae (used according to the manufacturer's instructions; Biomérieux). The strains were also typed by using PRA, as described by Telenti et al. (1993). Briefly, a region of DNA (439 bp) corresponding to the gene encoding Hsp65 was amplified by PCR and then digested with HaeIII or BstEIII; the fragments obtained were separated by agarose-gel electrophoresis and visualized under UV.

Extraction and analysis of fatty acids, mycolic acids and alcohols. Two to three loopfuls of bacteria (20-30 mg wet wt of each strain) were collected from the surface of the Sauton's agar plates. Fatty acids, mycolic acids and alcohols were liberated from each strain sample by saponification. These compounds were then extracted with diethyl ether. For each strain, an aliquot of the extract was treated with diazomethane to obtain the methyl esters of the fatty acids and mycolic acids (Daffé et al., 1983); in another aliquot of the extract the mycolic acids were transformed to $p$-bromophenacyl derivatives (Butler et al., 1991).

The $p$-bromophenacyl derivatives of the mycolic acids were separated in a HPLC system (Waters Associates) equipped with an UV/visible detector. A reverse-phase C18 column (Nova-Pack 60A, $4 \mu \mathrm{m}, 3.9 \times 75 \mathrm{~mm}$; Waters Associates) was used in the system; the mycolic acids were eluted using a linear gradient of methanol/chloroform [from 98:2(v/v) to $30: 70$ $(\mathrm{v} / \mathrm{v})]$. A high-molecular-mass standard (Ribi; ImmunoChem Research) was used as an internal standard, to assist in the identification of the peaks. Pattern-recognition software (PIROUETTE; Infometrix) was employed to evaluate the similarity of the HPLC chromatograms obtained, and was used in conjunction with a library containing the chromatograms of the most relevant Mycobacterium species and related bacteria (Glickman et al., 1994). Designation of HPLC peaks followed the arbitrary nomenclature used by several authors in the literature (Glickman et al., 1994).

Mycolic acid methyl esters were studied by analytical onedimensional TLC using silica gel 60 TLC plates (Merck). A triple development with a mixture of $n$-hexane/diethyl ether $(85: 15, \mathrm{v} / \mathrm{v})$ was performed to separate the individual mycolates. The components separated by TLC were revealed as dark-blue spots by spraying the TLC plates with $10 \%(\mathrm{w} / \mathrm{v})$ molybdophosphoric acid (Merck) in ethanol and heating them at $120^{\circ} \mathrm{C}$ for $5 \mathrm{~min}$. The methyl mycolates on the TLC plates were identified by comparing their positions on the plates with the mycolate patterns of reference strains (Luquin et al., 1991).

Fatty acid methyl esters, alcohols and methyl mycolate cleavage products were determined by GLC and GLC-MS as described previously (Luquin et al., 1991), employing a fusedsilica capillary column (cross-linked methyl silicone, $15 \mathrm{~m} \times$ $0.25 \mathrm{~mm}, \mathrm{HP}-1$; Hewlett Packard) that was programmed from 175 to $300{ }^{\circ} \mathrm{C}$ at $8{ }^{\circ} \mathrm{C} \mathrm{min}{ }^{-1}$ and maintained at $300{ }^{\circ} \mathrm{C}$ for $15 \mathrm{~min}$.

Purification and structural analysis of mycolates. Crude methyl mycolates from M. gordonae strains ATCC $14470^{\mathrm{T}}$, ATCC 35759 and CL-416C were obtained by precipitating their lipidic extracts with cold methanol $\left(-20^{\circ} \mathrm{C}\right.$, overnight). The samples were then centrifuged at $1500 \mathrm{~g}$ for $30 \mathrm{~min}$ at $4{ }^{\circ} \mathrm{C}$. Unless indicated otherwise, the boiling point of the petroleum ether used in the following procedures was 60 
$80{ }^{\circ} \mathrm{C}$. For purification of the methyl mycolates, the precipitates were recovered, dissolved in the smallest possible volume of petroleum ether and applied to a silica gel 60 (Merck; particle size 0.063-0.200 $\mu \mathrm{m}$ ) column equilibrated in petroleum ether. Successive elutions, 3-5 bed-volumes each, were performed with petroleum ether followed by increasing concentrations of diethyl ether $(5,10,20$ and $100 \%)$ in petroleum ether. The eluates were separated by TLC, as described above. Dicarboxy-mycolates were obtained in the $20 \%(\mathrm{v} / \mathrm{v})$ diethyl ether/petroleum ether fraction.

Purified mycolic acid methyl esters were identified by MS and NMR. Electron-impact MS (EI-MS) $(70 \mathrm{eV})$ and fast-atom bombardment MS (FAB-MS) $(8 \mathrm{eV})$ were performed in a VG AutoSpec (Fison) mass spectrometer. FAB-MS was carried out in the positive mode, employing $m$-nitrobenzyl alcohol as the matrix. Because FAB-MS seemed to produce $(\mathrm{M}+\mathrm{Na})^{+} / z$ pseudomolecular ions, we doped the matrix with $\mathrm{NaCl}$ in some analyses. Dicarboxy-mycolates from strain CL-416C were also analysed by FAB-MS in the negative mode and by chemical ionization (methane; temperature of ionization, $180^{\circ} \mathrm{C}$ ) in the negative mode. For the latter method of analysis, a Thermoquest-Trace mass spectrometer (Thermo) with a temperature range of $60-300{ }^{\circ} \mathrm{C}$ was used.

All methyl mycolates from strains ATCC $14470^{\mathrm{T}}$, ATCC 35759 and CL-416C were analysed by ${ }^{1} \mathrm{H}-\mathrm{NMR}$ at $300 \mathrm{MHz}$ in a Varian NMR spectrometer. Dicarboxy-mycolates from strains ATCC 35759 and CL-416C were also studied by ${ }^{13} \mathrm{C}$ NMR $(75 \mathrm{MHz})$ in the same spectrometer. In all cases, the spectra were recorded in deuterochloroform $[10 \mathrm{mg}(\mathrm{ml}$ sample $)^{-1}$ ] at $25^{\circ} \mathrm{C}$.

\section{RESULTS}

\section{Biochemical and genetic identification}

The five strains of clinical origin used in this study were identified as M. gordonae by standard biochemical tests and by Gen-Probe. Upon PRA, four of the clinical strains (CR-178, CL-361, CL-416C and CL-554) exhibited PRA pattern $\mathrm{V}$, as defined by Telenti et al. (1993); CL-333 exhibited PRA pattern III. M. gordonae ATCC 35759 exhibited pattern III and M. gordonae ATCC $14470^{T}$ exhibited pattern I.

\section{Mycolate chromatographic patterns}

Upon HPLC analysis of their mycolates, the five clinical strains of M. gordonae and strain ATCC 35759 showed an HPLC-double-cluster pattern (Cage, 1992) (Fig. 1a). TLC analysis of the mycolates revealed that the six strains contained $\alpha$-, methoxy-, keto-mycolates and additional components, such as secondary alcohols and dicarboxy-mycolates (Fig. 1b). M. gordonae ATCC $14470^{\mathrm{T}}$ showed the most common HPLC-single-cluster pattern (Cage, 1992) (data not shown); TLC revealed this strain to contain only $\alpha$, methoxy- and ketomycolates in its cell wall (Fig. 1b; lane 4). HPLC of the $p$-bromophenacyl derivatives of the total mycolic acids from M. gordonae CL-416C are shown in Fig. 1(a). As further demonstrated by HPLC (data not shown), dicarboxy-mycolates purified from strains ATCC 35759 and CL-416C and transformed to their p-bromophenacyl derivatives eluted as three peaks, which

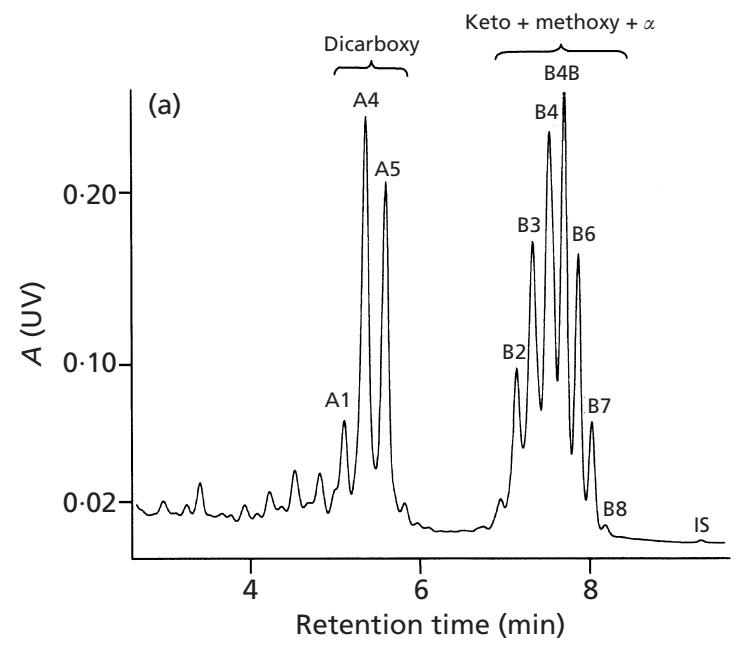

(b)

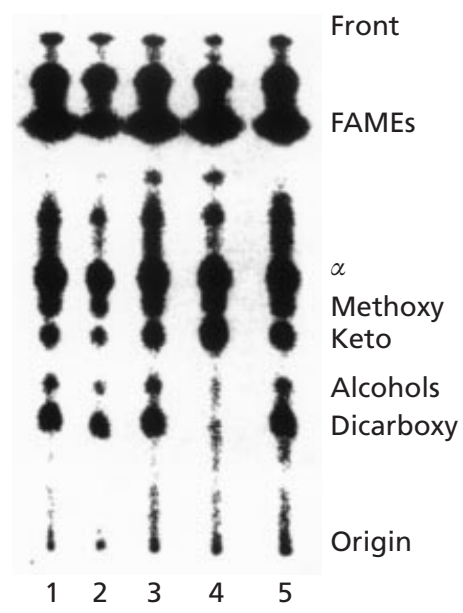

Fig. 1. (a) HPLC trace of mycolates from $M$. gordonae CL-416C, showing a double-cluster pattern. Nomenclature of the peaks is arbitrary but its correspondence to the different structural types of mycolates is given. IS, internal standard. (b) TLC of mycolic acids of different strains of $M$. gordonae. Lanes: $1, \mathrm{CR}-$ 178; 2, CL-416C; 3, CL-554; 4, ATCC 14470'; 5, CL-333. Petroleum ether/diethyl ether $(85: 15, v / v)$ was used as the solvent, twice. The b.p. of the petroleum ether was $60-80^{\circ} \mathrm{C}$. The different structural types of mycolates $(\alpha$, methoxy and keto) are indicated. Alcohols, secondary alcohols 2-octadecanol and 2-eicosanol; FAMEs, fatty acid methyl esters. Compounds migrating above the $\alpha$-mycolates probably correspond to epimerization products of the different mycolates.

corresponded exactly to peaks A1, A4 and A5 in Fig. 1 (a). Other components detected in the chromatogram, peaks B2-B8 (Glickman et al., 1994), corresponded to a mixture of keto-, methoxy- and $\alpha$-mycolates (Fig. 1a).

\section{Fatty acids and alcohols}

GLC analysis (data not shown) of the fatty acids of the clinical strains and of strains ATCC 35759 and ATCC $14470^{\mathrm{T}}$ indicated the presence of fatty acids with 14 to 24 carbon atoms in these strains, of which hexadecanoate and octadecenoate were the most prominent. Minor amounts of 2-methyl tetradecanoic acid were detected in 


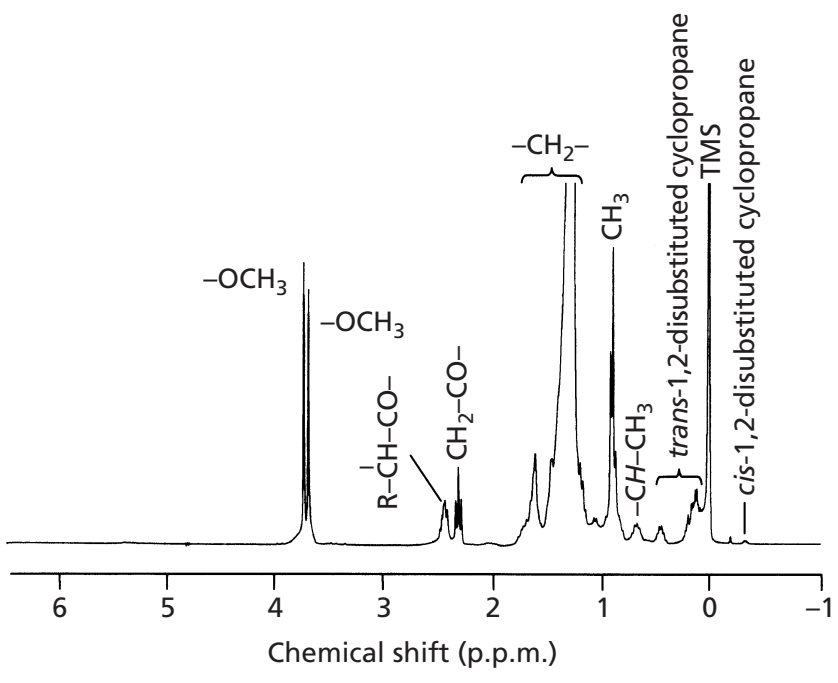

Fig. 2. ${ }^{1} \mathrm{H}-\mathrm{NMR}$ spectrum ( $300 \mathrm{MHz}$ ) of dicarboxy-mycolates from $M$. gordonae ATCC 35759 in deuterochloroform. Resonances of the different protons are indicated. TMS, tetramethylsilane.

the strains, whereas tuberculostearic acid (10-methyl octadecanoic acid) was not present in any of the strains examined. Two secondary alcohols, 2-octadecanol and 2-eicosanol, were detected only in those strains showing the HPLC-double-cluster pattern. The methyl esters derived from thermal cleavage of the methyl mycolates (Luquin et al., 1991) were docosanoate and tetracosanoate, which were present in varying amounts.

\section{Structures of the dicarboxy-mycolates of M. gordonae}

In the ${ }^{1} \mathrm{H}-\mathrm{NMR}$ spectrum of strain ATCC 35759 (Fig. 2), major resonances were detected between $0 \cdot 1$ and 3.7 p.p.m. A multiplet centred at 0.15 p.p.m. $(3 \mathrm{H})$ and another one at 0.47 p.p.m. $(1 \mathrm{H})$ were attributed to a trans-1,2-disubstituted cyclopropane ring (Draper et al., 1982; Watanabe et al., 1999). A signal at 0.67 p.p.m. (Fig. 2) was assigned to a $-\mathrm{CH}-\mathrm{CH}_{3}$ adjacent to the cyclopropane ring (Watanabe et al., 1999); the protons of the methyl branch resonated as a doublet at 0.90 p.p.m., and overlapped a signal of a terminal methyl at 0.88 p.p.m. (6H in total) (Draper et al., 1982). Singlets at 3.66 and 3.70 p.p.m. corresponded to two carbomethoxy groups, and the triplet at $2 \cdot 29$ p.p.m. was assigned to a $-\mathrm{CH}_{2}-$ attached to a carbonyl group. The protons of other $-\mathrm{CH}_{2}-$ groups resonated between $1 \cdot 2$ and 1.6 p.p.m. Finally, the signal at 2.42 p.p.m. was due to the proton of $\mathrm{C}-2$ of the molecule. Minor resonances situated at -0.3 and 0.73 p.p.m. (Fig. 2) were due to the presence of low amounts of a compound with a cis-1,2disubstituted cyclopropane ring (Watanabe et al., 1999).

In the ${ }^{13} \mathrm{C}-\mathrm{NMR}$ spectrum of strain ATCC 35759 (data not shown), two signals at $174 \cdot 30$ and $176 \cdot 19$ p.p.m. indicated the existence of two carbonyl groups in the molecule, which were complemented to the resonances of two carbomethoxy groups at 50.98 and 51.46 p.p.m. Resonances at $10.50,18 \cdot 62$ and $26 \cdot 14$ p.p.m. were assigned to a trans-1,2-disubstituted cyclopropane ring; the resonances situated at 19.70 and 38.13 p.p.m. were assigned to a methyl branch and to a methyne, respectively, which were both adjacent to the aforementioned ring (Watanabe et al., 1999). The presence of a hydroxyl group at C-3 was justified by the resonance at $72 \cdot 29$ p.p.m.

These data indicate that the additional component detected by TLC and HPLC in the M. gordonae clinical strains and in strain ATTC 35759 can be identified as a dicarboxy-mycolate (dimethyl ester form) that contains predominantly a trans-1,2-disubstituted cyclopropane ring with a methyl branch adjacent to it (Fig. 3; Table 1).

EI-MS (Fig. 3) revealed fragments related to the general breakdown (between C-2 and C-3) pattern of mycolates, which produce meroaldehydes and methyl esters (Draper et al., 1982). Methyl esters appeared at $m / z 382$ (tetracosanoate, $\mathrm{C}_{24: 0}$ ) and 354 (docosanoate, $\mathrm{C}_{22: 0}$ ). Fragments due to $M^{+}-50 / z$ (water and methanol loss) were detected at $m / z 908,936$ (the most predominant) and 964 , which predicted molecular ions $\left(\mathrm{M}^{+} / z\right)$ at 958 , 986 and 1014; these were further confirmed by positive-mode FAB-MS, because pseudomolecular ions, $(M+N a)^{+} / z$, appeared at 981, 1009 and 1037 (data not shown).

In the region of the EI-MS trace attributed to meroaldehydes (Fig. 3), a major fragment at $\mathrm{m} / z 601$ was accompanied by another one at $m / z 632$; other fragments were present at lower intensities. A formula for dicarboxy-mycolates was not satisfactory when $m / z 601$ was considered. However, negative-mode FAB-MS (data not shown) revealed very low intensities for pseudomolecular ions, $(M-H)^{-1} / z$, but important fragments at $(M-15) / z$, which were tentatively assigned to the loss of $-\mathrm{CH}_{3}$ from a carbomethoxy group. The elimination of a methyl group to give a negatively charged molecule (a carboxylate ion) could apparently take place in the meroaldehyde part of the structure, because intense fragments at $m / z 381$ and 353 (similar intensities) were clearly related to methyl esters derived from a breakdown between C-2 and C-3 of the molecule. Thus, an intense fragment at $m / z 617$ was detected by negative-mode FAB-MS and was considered to be related to a meroaldehyde at $m / z 632$ seen in the EI-MS analysis. Finally, by chemical ionization an important fragment (relative abundance $90 \%$ ) was detected at $\mathrm{m} / \mathrm{z}$ 631 (data not shown). Thus, it was concluded that the major meroaldehyde produced by the compound under study was actually $m / z 632$. Moreover, this fragment satisfied the formula given in Fig. 3, when an $n 2+n 3$ value equal to 34 was considered. Then, the meroaldehyde at $m / z 632$ fragments to $m / z 601$ due to loss of $-\mathrm{OCH}_{3}$ (Fig. 3a), because of the existence of the second ester group at the $\omega$ end. Similarly, an $m / z 573$ (Fig. 3a) was derived from a meroaldehyde at $m / z 604$ (very low intensity) ( $n 2+n 3$ equal to 32$)$.

The value of $n 3$ was deduced by taking into account the 


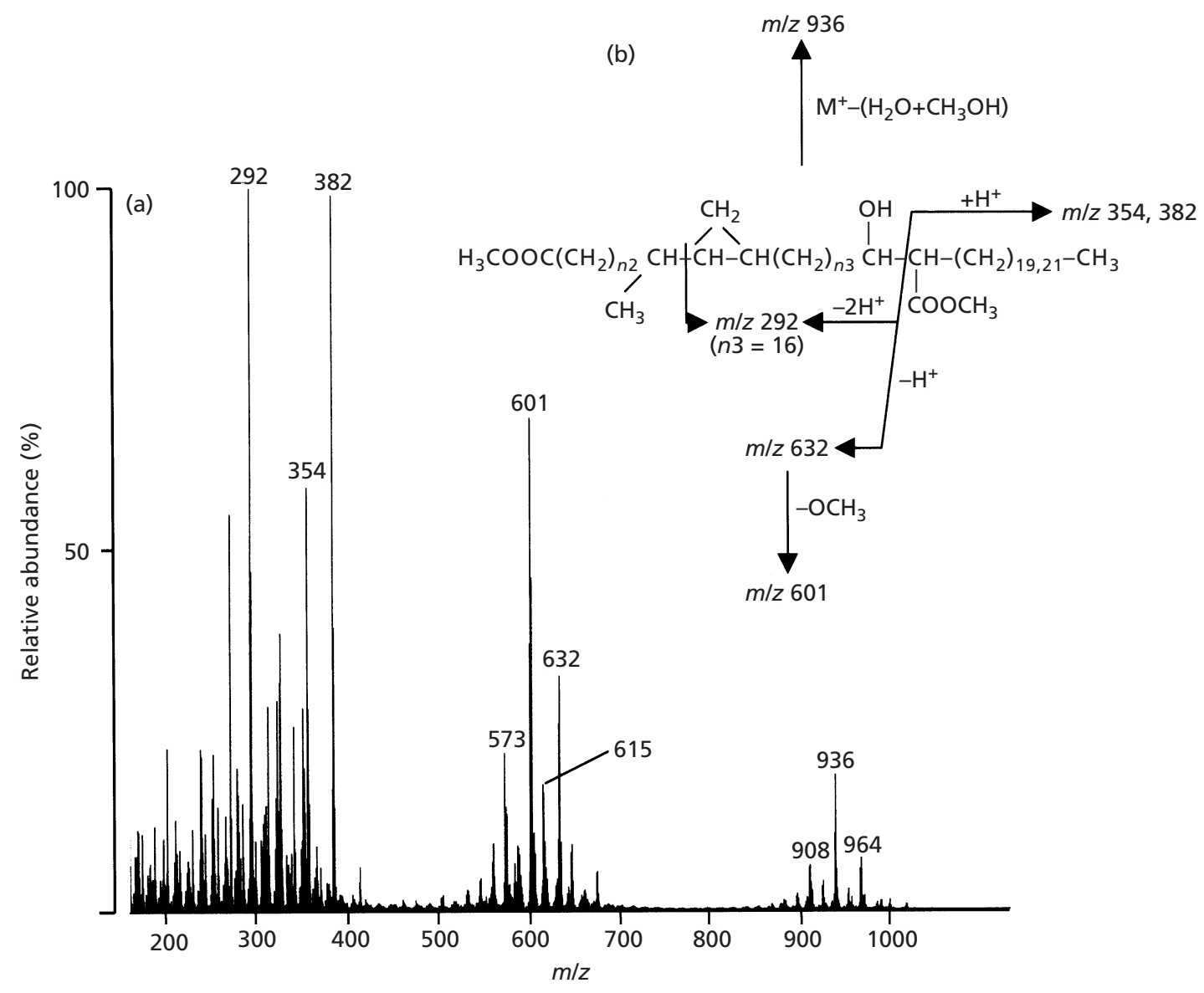

Fig. 3. (a) Partial EI-MS trace of dicarboxy-mycolates from M. gordonae ATCC 35759. (b) The structure and the general fragmentation pattern of the major component are included, showing the $\mathrm{m} / \mathrm{z}$ ions corresponding to meroaldehydes ( $\mathrm{m} / \mathrm{z}$ 632, 601 and 292) methyl esters ( $\mathrm{m} / \mathrm{z} 354$ and 382) and $M^{+}-50 / z(\mathrm{~m} / \mathrm{z} 936$; loss of water and methanol).

presence of a very intense fragment at $m / z 292$ in the EI-MS analysis (Fig. 3a), which was derived from a breakdown between the cyclopropane ring and the carbon attached to the methyl branch and a further rearrangement. This fragment should indicate that for $m / z 632, n 3$ is equal to 16 and $n 2$ is equal to 18 , and suggested that the major dicarboxy-mycolate from $M$. gordonae contains 63 carbon atoms, with an alkyl chain at $\mathrm{C}-2$ of 20 carbon atoms (producing a $\mathrm{C}_{22: 0}$ methyl ester). For a meroaldehyde at $m / z 604(n 2=16, n 3=16)$, a compound with a molecular mass of 986 is also obtained assuming an alkyl chain of 22 carbon atoms at $\mathrm{C}-2$ (producing a $\mathrm{C}_{24: 0}$ methyl ester), thus justifying a more intense peak at $m / z 936\left(M^{+}-50\right)$ (see above and Fig. 3) and at $(M+N a)^{+} / z 1009$ (data not shown).

The two remaining homologous compounds seen in the EI-MS analysis can also be formulated in a similar way. Thus, a $\mathrm{C}_{65}$ dicarboxy-mycolate with a molecular mass of 1014 is composed combining the $\mathrm{m} / z 632$ meroaldehyde and the $m / z 382$ methyl ester; and a $\mathrm{C}_{61}$ dicarboxy-mycolate (molecular mass 958) is composed by combining $m / z 604$ (a meroaldehyde) and $m / z 354$ (a methyl ester).
Both ${ }^{1} \mathrm{H}-\mathrm{NMR}$ (Fig. 2) and MS [EI-MS (Fig. 3) and FAB-MS (data not shown)] predicted the presence of minor amounts of dicarboxy-mycolates with a cis-1,2disubstituted-cyclopropane ring in the strains producing a HPLC-double-cluster pattern. The major meroaldehyde for this series was detected at $\mathrm{m} / \mathrm{z} 646$ (fragment at $m / z 615$; Fig. 3a), and the chain length varied between $\mathrm{C}_{60}$ and $\mathrm{C}_{64}$ according to the MS: pseudomolecular ions, $(M+N a)^{+} / z$, were situated at $967\left(\mathrm{C}_{60}\right), 995\left(\mathrm{C}_{62}\right.$, probably the predominant pseudomolecular ion) and $1023\left(\mathrm{C}_{64}\right)$ (data not shown).

\section{Structures of the other mycolates of $\boldsymbol{M}$. gordonae}

The structures of the remaining mycolates $(\alpha-$, methoxyand keto-mycolates) of strains ATCC 35759, CL-416C and ATCC $14470^{\mathrm{T}}$ were established by ${ }^{1} \mathrm{H}-\mathrm{NMR}$ and MS (EI-MS and positive-mode FAB-MS); the results of these analyses are presented in Table 1.

$\alpha$-Mycolates from M. gordonae strains ATCC 35759 and CL-416C contained mainly two cis-1,2-disubstituted cyclopropane rings (their protons were located at $-0.32,0.59$ and 0.64 p.p.m.), and even chain 
Table 1. Structural characteristics of the major mycolates of strains of M. gordonae

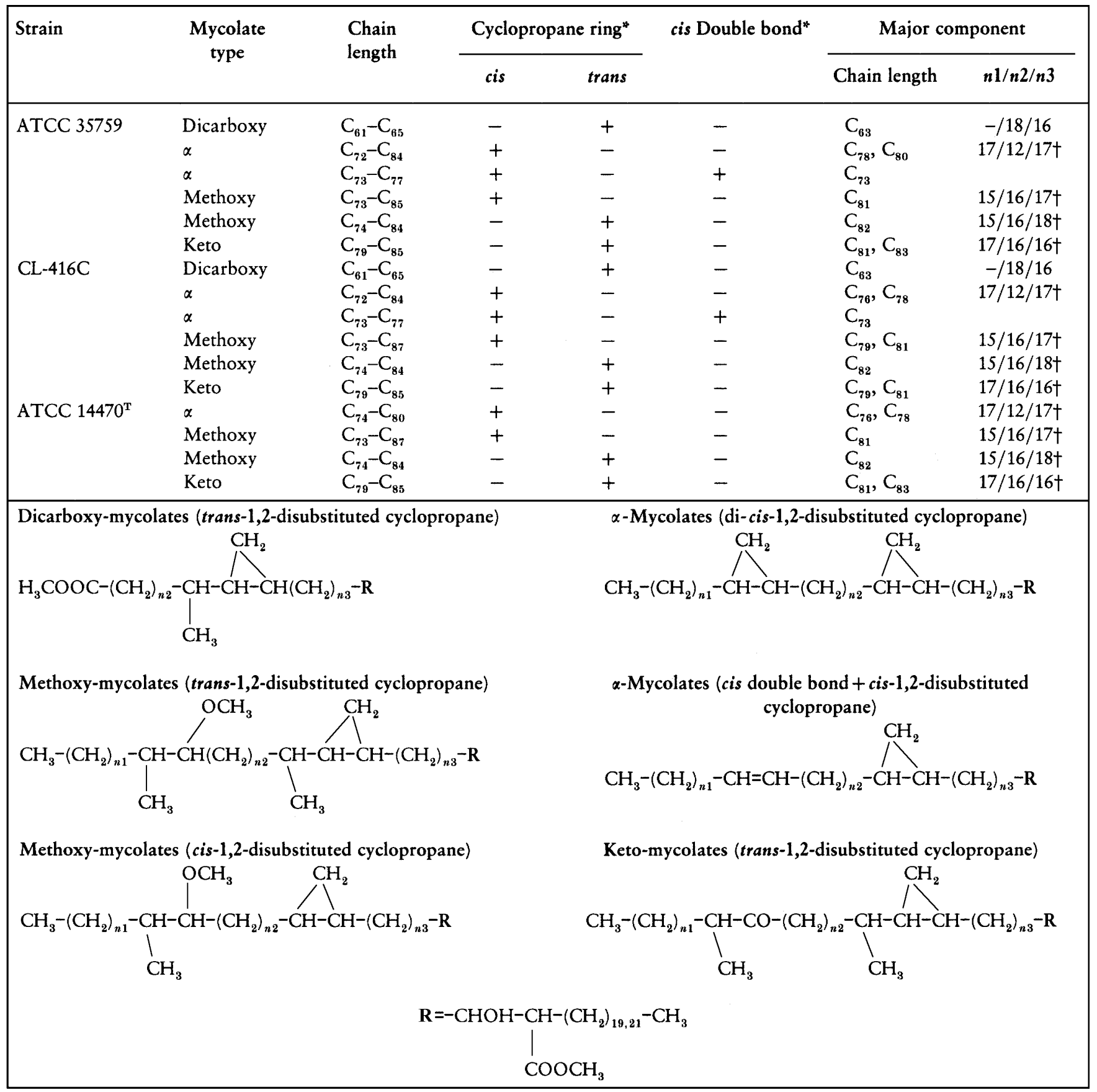

*-, Absent; +, present.

†The given values are only tentative.

lengths of $\mathrm{C}_{72}-\mathrm{C}_{84}$ (major series at $\mathrm{C}_{76}$ and $\mathrm{C}_{78}$ for CL$416 \mathrm{C}$, and at $\mathrm{C}_{78}$ and $\mathrm{C}_{80}$ for ATCC 35759). Major meroaldehyde ions $(m / z 740,768$ and 796) and their fragments $(m / z 279,307,487,515$ and 543), together with methyl ester fragments at $m / z 354$ and 382, permitted the tentative structures in Table 1 to be proposed (Draper et al., 1982). Moreover, other compounds probably containing one cis double bond (resonance at 5.34 p.p.m.) and one cis-1,2-disubstituted cyclopropane ring were also predicted in the two aforementioned strains; both EI-MS and FAB-MS signalled chain lengths of $\mathrm{C}_{73}-\mathrm{C}_{77}$. The ratio of $-\mathrm{CH}=\mathrm{CH}-$ protons to 1,2-disubstituted cyclopropane ring protons was 21 and 22.7 for strains ATCC 35759 and CL-416C, respectively, implying that these compounds could represent approximately $20 \%$ of the total amount of $\alpha$ mycolates present in these strains. Finally, M. gordonae ATCC $14470^{\mathrm{T}}$ contained $\alpha$-mycolates with only two cis- 
1,2-disubstituted cyclopropane rings, which ranged from $\mathrm{C}_{74}$ to $\mathrm{C}_{80}$ (major compounds $\mathrm{C}_{76}$ and $\mathrm{C}_{78}$ ).

The methoxy-mycolates of strains ATCC 35759 , CL416C and ATCC $14470^{\mathrm{T}}$ were identical (Table 1). They were clearly identified by ${ }^{1} \mathrm{H}-\mathrm{NMR}$ due to characteristic overlapping signals at 0.85 (a methyl branch adjacent to the methoxy group of the meroaldehyde chain), $0 \cdot 88$ (a terminal methyl group) and 0.90 p.p.m. (a methyl branch adjacent to a 1,2-disubstituted cyclopropane ring), and also by signals at $2.95\left(-\mathrm{CH}^{-} \mathrm{OCH}_{3}\right)$ and 3.33 p.p.m. $\left(-\mathrm{OCH}_{3}\right)$. Methoxy-mycolates containing cis- $(-0 \cdot 32$, 0.59 and 0.64 p.p.m.) or trans- $(0.15$ and 0.47 p.p.m.) 1,2-disubstituted cyclopropane series of $\mathrm{C}_{73}-\mathrm{C}_{87}$ were detected, with series of $\mathrm{C}_{81}$ and $\mathrm{C}_{82}$ predominant for strains ATCC 35759 and ATCC $14470^{\mathrm{T}}$, and series of $\mathrm{C}_{81}$ and $\mathrm{C}_{79}$ predominant for strain CL-416C. The ratio of cis to trans was approximately $1: 1$, and given the structure of methoxy-mycolates of other mycobacteria (Minnikin, 1982; Watanabe et al., 2001), it seems that the odd series can be attributed to cis compounds and the even series can be attributed to trans compounds. Meroaldehydes from methoxy-mycolates were not found in EI-MS due, probably, to the loss of 31 mass units $\left(-\mathrm{OCH}_{3}\right)$, which resulted in a series of $m / z$ ions ranging from 769 to 867 (separated by intervals of 14 mass units) that predicted meroaldehydes at $\mathrm{m} / z$ 800-898. Other fragments characteristic of meroaldehydes were located at $m / z 297$ (intensity 72\%), 307 (intensity $19 \%$ ) and 321 (intensity 16\%); considering the $m / z 354$ and 382 for methyl esters, the tentative structure presented in Table 1 can be postulated for the major compounds found in the strains studied.

Keto-mycolates (Table 1) were characterized by resonances at 0.90 (a methyl branch adjacent to a 1,2disubstituted cyclopropane ring), 1.05 (a methyl branch adjacent to the keto group in the meroaldehyde chain) and $2 \cdot 32$ p.p.m. $\left(-\mathrm{CH}_{2}-\mathrm{CO}-\right)$. The major series varied from $\mathrm{C}_{79}$ to $\mathrm{C}_{85}$ and had one trans-1,2-disubstituted cyclopropane ring in the structure (resonances at 0.15 and 0.47 p.p.m.). $\mathrm{C}_{81}$ and $\mathrm{C}_{83}$, were the predominant series for strains ATCC $14470^{\mathrm{T}}$ and ATCC 35759 ; $\mathrm{C}_{81}$ and $\mathrm{C}_{79}$ were the predominant series for strain CL-416C. FAB-MS also predicted very minor series of mycolates with a chain length of $\mathrm{C}_{78}-\mathrm{C}_{80}$ in the three aforementioned strains, probably containing a cis-1,2disubstituted cyclopropane ring, as suggested by ${ }^{1} \mathrm{H}$ NMR (data not shown). The tentative structure of the major keto-mycolate series is given in Table 1 and was based on the presence of important fragments at $\mathrm{m} / z$ 293, 491, 547 and 601 (derived from meroaldehydes), and at $m / z 354$ and 382 (methyl esters).

\section{DISCUSSION}

Analyses of mycolic acids are well established in mycobacterial taxonomy. Extensive studies have described mycolic acids as phenotypically stable chemical compounds, although the discontinuous distribution of the different structural types of mycolic acids described has important chemotaxonomic implications. Since the 1950s, GLC and TLC have been the chromatographic techniques most widely used to analyse the composition of mycobacterial mycolic acids as methyl esters (Daffé et al., 1983; Minnikin et al., 1984; Luquin et al., 1991; Valero-Guillén et al., 1985). In the 1990s, phenacyl esters of mycolic acids were separated by HPLC and detected under UV light (Butler \& Guthertz, 2001). The strainidentification data generated by HPLC agree with $97 \%$ of the results from standard biochemical tests and $98 \%$ of the results from DNA probes; hence, HPLC can be used to correctly identify the majority of mycobacterial species of clinical interest (Butler \& Guthertz, 2001; Thibert \& Lapierre, 1993).

The elucidation of the structures of mycolic acids uses a variety of techniques (Minnikin, 1982), the most widely used of which are EI-MS and NMR. Recently, new techniques, such as matrix-assisted laser desorption ionization/time-of-flight MS (MALDI/TOF-MS) (Laval et al., 2001; Watanabe et al., 2001) and FAB-MS (Barry et al., 1998), have been applied to the elucidation of these structures. In this study, FAB-MS data complemented the data obtained by EI-MS, giving more precise information on the molecular mass of the different structural types of mycolic acids present in M. gordonae. This information is not always evident when using EI-MS, as there is a tendency for several meroaldehydes (e.g. methoxy and dicarboxy) to break down further due to loss of $-\mathrm{OCH}_{3}$.

Using HPLC, Cage (1992) demonstrated two different mycolic acid patterns for M. gordonae - a single-cluster pattern and a double-cluster pattern. In the present study, we have demonstrated that the second cluster of peaks present in the double-cluster pattern actually corresponds to dicarboxy-mycolates, and that these compounds essentially contain a trans-1,2-disubstituted cyclopropane ring, although a minor series with a cis1,2-disubstituted cyclopropane has also been detected. As reported by Lanéelle \& Lanéelle (1970), dicarboxymycolates and secondary alcohols (2-eicosanol and 2octadecanol) are naturally combined in the cell wall to form wax-ester mycolates. These compounds are widely distributed in mycobacteria (Barry et al., 1998; Luquin et al., 1991; Minnikin et al., 1984, 1985a), but only a limited number of studies have dealt with their structural elucidation. Thus, the dicarboxy-mycolates of Mycobacterium phlei (see Minnikin, 1982) are mixtures of unsaturated and cyclopropyl homologues, and differ from those found in M. gordonae, in which only cyclopropyl derivatives were detected.

Taking into account the presence of 2-octadecanol and 2-eicosanol in the M. gordonae strains containing dicarboxy-mycolates, the chain length of wax-ester mycolates from M. gordonae should range from $\mathrm{C}_{81}$ to $\mathrm{C}_{85}$ for the principal components, which is similar to the chain lengths detected for the principal keto-mycolates. Like the keto-mycolates, the major series of the waxester mycolates contain a trans-1,2-disubstituted cyclopropane ring, thus supporting the hypothesis that ketoand wax-ester mycolates are biosynthetically related (Minnikin, 1982; Barry et al., 1998). 
Partial structural analyses of $\alpha$-, methoxy- and ketomycolates from $M$. gordonae have been reported previously (Daffé et al., 1981; Minnikin et al., 1985b). However, for the first time, our work has established the nature of the double bonds and the cyclopropyl rings in this species. Thus, $\alpha$-mycolates from $M$. gordonae are mostly of type-1, as defined by Watanabe et al. (2001) i.e. they contain two cyclopropyl rings with no double bonds. Most slow-growing mycobacterial species examined to date (Minnikin, 1982; Watanabe et al., 2001) contain predominantly this type of $\alpha$-mycolate, with a cis to trans ratio of 1:0 (Watanabe et al., 2001), as in the case of M. gordonae, although minor series of transcyclopropyl $\alpha$-mycolates have been found in $\mathrm{Myco-}$ bacterium kansasii and in the Mycobacterium avium complex (Watanabe et al., 2001). However, other types of $\alpha$-mycolates have been defined in several mycobacterial species (Watanabe et al., 2001), but only the so-called type-3 $\alpha$-mycolate (one cis-cyclopropyl ring plus one cis double bond) seems to be present in the $M$. gordonae strains containing dicarboxy-mycolates (this study). This type of $\alpha$-mycolate has also been characterized in several strains of Mycobacterium tuberculosis, Mycobacterium bovis and Mycobacterium microti and in strains of the $M$. avium complex (Watanabe et al., 2001). The aforementioned species also contain a variety of methoxy-mycolates, the major ones being of the type methoxy-mycolate- 1 (Watanabe et al., 2001) (one series with a cis-cyclopropyl ring and one series with a trans-cyclopropyl ring), a characteristic shared by Mycobacterium leprae (Draper et al., 1982) and, according to our results, by M. gordonae. As for its $\alpha$-mycolates, $M$. gordonae seems to lack trans double bonds or additional cyclopropyl rings in the meroaldehyde chain and, contrary to other mycobacteria (Watanabe et al., 2001), it lacks methoxy-mycolates with cis double bonds. Among the keto-mycolates of M. gordonae, we only detected the keto-mycolate-1 (Watanabe et al., 2001), generally with a transcyclopropyl ring. Hence, the keto-mycolates of M. gordonae resemble those of species such as M. kansasii and M. avium, and differ from those of the M. tuberculosis complex, where, in general, the cis to trans ratio is more balanced (Watanabe et al., 2001).

According to the results cited above, it seems that a high proportion of the mycolic acids of $M$. gordonae have trans cyclopropanation, but like other mycobacteria (Barry et al., 1998; Minnikin, 1982; Watanabe et al., 2001) M. gordonae still maintains a cis configuration in the cyclopropyls of its $\alpha$-mycolates. An appropriate ratio in the cis to trans geometry of both double bonds and cyclopropyls seems to have physiological significance for the bacterial cell wall, but its true relevance remains to be elucidated (Barry et al., 1998).

The M. gordonae clinical strains studied here exhibit PRA patterns III and V, which are only present in $12 \%$ of M. gordonae isolates (Telenti et al., 1993). The mycolic acid pattern exhibited by these clinical isolates is also uncommon in members of the genus Mycobacterium, as the presence of $\alpha$-, methoxy-, keto- and dicarboxy-mycolates has only been reported in two additional species of the genus, Mycobacterium komossense and Mycobacterium bohemicum (Minnikin et al., 1985a; Torkko et al., 2001).

We conclude that the variability in the mycolic acid patterns of strains of M. gordonae, as detected by HPLC and TLC, exists because of the presence of additional components (dicarboxy-mycolates) in some strains of this species. These additional components give rise to the HPLC-double-cluster pattern. Hence, our findings reiterate the heterogeneity of $M$. gordonae reported previously by several authors (Kirschner \& Böttger, 1992; Plikaytis et al., 1992; Telenti et al., 1993; Walton \& Valesco, 1991).

\section{ACKNOWLEDGEMENTS}

The authors are grateful to Dr Gary D. Cage (Department of Health Services, Arizona, USA) for his kind collaboration in this work. The technical assistance of Lidia Hernández is gratefully acknowledged. We are also indebted to $\mathrm{Dr} \mathrm{J}$. Rodríguez (SACE, University of Murcia, Spain) for mass spectrometry analyses. This work was supported by grants PM98-0180, FIS 97/0584 and AYA 9800/01.

\section{REFERENCES}

Barry, C. E., 3rd, Lee, R. E., Mdluli, K., Sampson, A. E., Schroeder, B. G., Slayden, R. A. \& Yuan, Y. (1998). Mycolic acids: structure, biosynthesis and physiological functions. Prog Lipid Res 37, 143-179.

Bernard, E., Michiels, J. F., Pinier, Y., Bourdet, J. F. \& Dellamonica, P. (1992). Disseminated infection as a result of Mycobacterium gordonae in an AIDS patient. AIDS 6, 1217-1218.

Butler, W. R. \& Guthertz, L. S. (2001). Mycolic acid analysis by high-performance liquid chromatography for identification of Mycobacterium species. Clin Microbiol Rev 14, 704-726.

Butler, W. R., Jost, K. C., Jr \& Kilburn, J. O. (1991). Identification of mycobacteria by high-performance liquid chromatography. J Clin Microbiol 29, 2468-2472.

Cage, G. D. (1992). High-performance liquid chromatography patterns of Mycobacterium gordonae mycolic acids. J Clin Microbiol 30, 2402-2407.

Clague, H., Hopkins, C. A., Roberts, C. \& Jenkins, P. A. (1985). Pulmonary infection with Mycobacterium gordonae in the presence of bronchial carcinoma. Tubercle 66, 61-63.

Daffé, M., Lanéelle, M. A., Promé, D. \& Asselineau, C. (1981). Étude des lipides de Mycobacterium gordonae comparativement à ceux de $M$. leprae et de quelques mycobactéries scotochromogènes. Ann Microbiol (Paris) 132B, 3-12.

Daffé, M., Lanéelle, M. A., Asselineau, C., Lévy-Frebault, V. \& David, H. (1983). Intérêt taxonomique des acides gras des mycobactéries: proposition d'une methode d'analyse. Ann Microbiol (Paris) 134B, 241-256.

Draper, P., Dobson, G., Minnikin, D. E. \& Minnikin, S. M. (1982). The mycolic acids of Mycobacterium leprae harvested from experimentally infected nine-banded armadillos. Ann Microbiol (Paris) 133, 39-47.

Glickman, S. E., Kilburn, J. O., Butler, W. R. \& Ramos, L. S. (1994). Rapid identification of mycolic acid patterns of mycobacteria by high-performance liquid chromatography using pattern recognition software and a Mycobacterium library. J Clin Microbiol 32, 740-745. 
Kirschner, P. \& Böttger, E. C. (1992). Microheterogeneity within rRNA of Mycobacterium gordonae. J Clin Microbiol 30, 1049-1050.

Lanéelle, M. A. \& Lanéelle, G. (1970). Structure d'acides mycoliques et d'un intermediaire dans la biosynthèse d'acides mycoliques dicarboxyliques. Eur J Biochem 12, 296-300.

Laval, F., Lanéelle, M. A., Déon, C., Monsarrat, B. \& Daffé, M. (2001). Accurate molecular mass determination of mycolic acids by MALDI-TOF mass spectrometry. Anal Chem 73, 4537-4544.

Le Dantec, C., Duguet, J.-P., Montiel, A., Dumoutier, N., Dubrou, S. \& Vincent, V. (2002). Chlorine disinfection of atypical mycobacteria isolated from a water distribution system. Appl Environ Microbiol 68, 1025-1032.

Leoni, E., Legnani, P., Mucci, M. T. \& Pirani, R. (1999). Prevalence of mycobacteria in a swimming pool environment. $J$ Appl Microbiol 87, 683-688.

London, R. D., Damsker, B., Neibart, E. P., Knorr, B. \& Bottone, E. J. (1988). Mycobacterium gordonae: an unusual peritoneal pathogen in a patient undergoing continuous ambulatory peritoneal dialysis. Am J Med 85, 703-704.

Luquin, M., Roussel, J., López-Calahorra, F., Lanéelle, G., Ausina, V. \& Lanéelle, M. A. (1990). A novel mycolic acid in a $\mathrm{Myco-}$ bacterium sp. from the environment. Eur J Biochem 192, 753-759.

Luquin, M., Ausina, V., López-Calahorra, F., Belda, F., GarcíaBarceló, M., Celma, C. \& Prats, G. (1991). Evaluation of practical chromatographic procedures for identification of clinical isolates of mycobacteria. J Clin Microbiol 29, 120-130.

Minnikin, D. E. (1982). Lipids: complex lipids, their chemistry, biosynthesis and roles. In The Biology of the Mycobacteria, vol. 1, pp. 95-184. Edited by C. Ratledge \& J. L. Stanford. London: Academic Press.

Minnikin, D. E., Minnikin, S. M. \& Goodfellow, M. (1982). The oxygenated mycolic acids of Mycobacterium fortuitum, M. farcinogenes and M. senegalense. Biochim Biophys Acta 712, 616-620.

Minnikin, D. E., Minnikin, S. M., Parlett, J. H., Goodfellow, M. \& Magnusson, M. (1984). Mycolic acid patterns of some species of Mycobacterium. Arch Microbiol 139, 225-231.

Minnikin, D. E., Minnikin, S. M., Parlett, J. H. \& Goodfellow, M. (1985a). Mycolic acid patterns of some rapidly-growing species of Mycobacterium. Zentralbl Bakteriol Mikrobiol Hyg Ser A 259, 446-460.

Minnikin, D. E., Dobson, G., Goodfellow, M., Draper, P. \& Magnusson, M. (1985b). Quantitative comparison of the mycolic and fatty acid compositions of Mycobacterium leprae and Mycobacterium gordonae. J Gen Microbiol 131, 2013-2021.

Papapetropoulou, M., Tsintzou, A. \& Vantarakis, A. (1997). Environmental mycobacteria in bottled table waters in Greece. Can J Microbiol 43, 499-502.
Plikaytis, B. B., Plikaytis, B. D., Yakrus, M. A., Butler, W. R., Woodley, C. L., Silcox, V. A. \& Shinnick, T. M. (1992). Differentiation of slowly growing Mycobacterium species including Mycobacterium tuberculosis, by gene amplification and restriction fragment length polymorphism analysis. J Clin Microbiol 30, 1815-1822.

Rusconi, S., Gori, A., Vago, L., Marchetti, G. \& Franzetti, F. (1997). Cutaneous infection caused by Mycobacterium gordonae in a human immunodeficiency virus-infected patient receiving antimycobacterial treatment. Clin Infect Dis 25, 1490-1491.

Telenti, A., Marchesi, F., Balz, M., Bally, F., Böttger, E. C. \& Bodmer, T. (1993). Rapid identification of mycobacteria to the species level by polymerase chain reaction and restriction enzyme analysis. J Clin Microbiol 31, 175-178.

Thibert, L. \& Lapierre, S. (1993). Routine application of highperformance liquid chromatography for identification of mycobacteria. J Clin Microbiol 31, 1759-1763.

Torkko, P., Suomalainen, S., livanainen, E. \& 7 other authors (2001). Characterization of Mycobacterium bohemicum isolated from human, veterinary, and environmental sources. J Clin Microbiol 39, 207-211.

Tsukamura, M. (1967). Identification of mycobacteria. Tubercle 48, 311-338.

Valero-Guillén, P. L., Pacheco, F. \& Martín-Luengo, F. (1985). Fatty acid composition and mycolic acid pattern of some chromogenic mycobacteria. J Appl Bacteriol 59, 113-126.

Vogiatzakis, E., Stefanou, S., Skroubelou, A., Anagnostou, S., Marinis, E. \& Matsiota-Bernard, P. (1998). Molecular markers for the investigation of Mycobacterium gordonae epidemics. J Hosp Infect 38, 217-222.

Walton, D. T. \& Valesco, M. (1991). Identification of Mycobacterium gordonae from culture by the Gen-Probe Rapid Diagnostic System: evaluation of 218 isolates and potential sources of false-negative results. J Clin Microbiol 29, 1850-1854.

Watanabe, M., Ohta, A., Sasaki, S. \& Minnikin, D. E. (1999). Structure of a new glycolipid from the Mycobacterium aviumMycobacterium intracellulare complex. J Bacteriol 181, 22932297.

Watanabe, M., Aoyagi, Y., Ridell, M. \& Minnikin, D. E. (2001). Separation and characterization of individual mycolic acids in representative mycobacteria. Microbiology 147, 1825-1837.

Wayne, L. G. \& Kubica, G. P. (1986). Genus Mycobacterium Lehmann and Neumann 1896, 363 ${ }^{\mathrm{AL}}$. In Bergey's Manual of Systematic Bacteriology, vol. 2, pp. 1436-1457. Edited by P. H. A. Sneath, N. S. Mair, M. E. Sharpe \& J. G. Holt. Baltimore, MD: Williams \& Wilkins.

Received 16 April 2002; revised 11 July 2002; accepted 12 July 2002. 